BULLETIN OF THE

AMERICAN MATHEMATICAL SOCIETY

Volume 77, Number 4, July 1971

\title{
SUBSTITUTION MINIMAL FLOWS ${ }^{1}$
}

\author{
BY JOHN C. MARTIN
}

Communicated by W. Gottschalk, February 25, 1971

We investigate the structure of a certain class of minimal symbolic flows (substitution minimal flows) which are natural generalizations of the widely studied Morse minimal set (see, for example, [3], [5]). We present here a brief description of the major results; detailed proofs will appear elsewhere. The author wishes to thank William Veech for his help in the preparation of this paper.

Let $S=\{0,1, \cdots, b-1\}$, and for $n \geqq 1$, let $S^{n}=\{f$ : $\{0,1, \cdots, n-1\} \rightarrow S\}$. If $A \in S^{n}$, we represent $A$ as $a_{0} \cdots a_{n-1}$, where $a_{i}=A(i)$; we refer to $A$ as an $n$-block (over $S$ ). For $A \in S^{n}$, $B \in S^{m}$, we let $A B=a_{0} a_{1} \cdots a_{n-1} b_{1} b_{2} \cdots b_{m-1}$, so that $A B \in S^{n+m}$. A substitution $\theta\left(=\theta^{1}\right)$ of length $r$ over $S$ is a map $\theta: S \rightarrow S^{r}$ with $\theta(0)(0)=0$. For $k \geqq 2$, if $\theta(j)=a_{0} a_{1} \cdots a_{r-1}$, we define $\theta^{k}(j)=\theta^{k-1}\left(a_{0}\right)$ $\cdots \theta^{k-1}\left(a_{r-1}\right)$. We define a sequence $x_{\theta}^{\prime}$ over $S$ by letting the $r^{k}$-block $x_{\theta}^{\prime}(0) x_{\theta}^{\prime}(1) \cdots x_{\theta}^{\prime}\left(r^{k}-1\right)$ be $\theta^{k}(0)$, for each $k \geqq 1$. $\theta$ is an admissible substitution if $\theta$ is one-to-one, range $x_{\theta}^{\prime}=S$, and $x_{\theta}^{\prime}$ is a recurrent, nonperiodic sequence. (It is not difficult to prescribe simple conditions which ensure that $\theta$ is admissible.) $\theta$ is simple, if for $i, j \in S$ $(i \neq j), \theta(i)(n) \neq \theta(j)(n)(0 \leqq n \leqq r-1)$. If $\theta$ is an admissible substitution, we choose any recurrent extension $x_{\theta}$ of $x_{\theta}^{\prime}$ to the integers, and we define $x_{\theta}=\left(X_{\theta}, T\right)$ to be the flow whose phase space $X_{\theta}$ is the orbit-closure of $x_{\theta}$ under the left shift $T$, in the space of all doubly infinite sequences over $S$ (with the product topology). $X_{\theta}$ is an infinite, compact metric space, and $x_{\theta}$ is a minimal flow. Finally, we obtain a positive integer $m(\theta)$ with $\operatorname{gcd}(m(\theta), r)=1$ so that $S$ is partitioned into nonempty sets $S_{0}, S_{1}, \cdots, S_{m(\theta)-1}$, and if $i \in S_{n(i)}(i \in S)$, the sequence of integers $n\left(x_{\theta}(j)\right)(j=0,1, \cdots)$ is periodic of period $m(\theta)$.

If $\theta$ is a fixed admissible substitution of length $r$ over $S$, our principal results may be stated as follows. Some of our results generalize certain results in [1] and [4]. (All definitions are as in [10].)

AMS 1969 subject classifications. Primary 5420.

Key words and phrases. Symbolic flow, Morse minimal set, substitution, equicontinuous structure relation, almost automorphic flow, almost automorphic extension, isometric extension, AI extension, AI flow, point-distal flow.

${ }^{1}$ Research partially supported by NSF Grant No. 8997. 
ThEOREM 1. $X_{\theta}$ is a point-distal flow with a residual set of distal points.

THEOREM 2. Let $\Sigma$ be the equicontinuous structure relation on $X_{0}$. Then $\mathfrak{X}_{\theta} / \Sigma$ is isomorphic to the equicontinuous flow $\left(\mathcal{Z}_{m(\theta)} \times \mathcal{Z}^{r}, T\right)$, where $\mathcal{Z}_{m(\theta)}$ is the cyclic group of order $m(\theta), Z^{r}$ is the $r$-adic completion of the integers, and $T$ is the homeomorphism determined by addition of the group element $(1,1)$.

COROllaRy. If $\theta$ is a binary substitution, $\mathfrak{X}_{\theta} / \Sigma=\left(\mathcal{Z}^{r}, T\right)$.

THEOREM 3. $x_{\theta}$ is an almost automorphic flow if and only if there exist integers $i, j, k\left(0 \leqq i \leqq m(\theta)-1, j \geqq 1,0 \leqq k \leqq r^{j}-1\right)$ with $\theta^{j}(p)(k)$ $=\theta^{j}(q)(k)$ for $p, q \in S_{i}$.

In [8], Veech represents the Morse flow (the substitution flow generated by the binary substitution $\theta(0)=01, \theta(1)=10$ ) as an isometric extension of an almost automorphic extension of $\left(\mathcal{Z}^{2}, T\right)$. This may be generalized in the following manner. We define $P_{\theta}=$ $\left\{x_{\theta}(j) x_{\theta}(j+1): j=0,1, \cdots\right\} \subset S^{2} ; A_{i j k}=\left\{\theta^{j}(p)(k) \theta^{j}(p)(k+1): p \in S_{i}\right\}$ $\subset P_{\theta}\left(0 \leqq i \leqq m(\theta)-1, j \geqq 1,0 \leqq k \leqq r^{j}-2\right)$.

THEOREM 4. If $\theta$ is simple, $x_{\theta}$ is an AI extension (i.e., an isometric extension of an almost automorphic extension) of an equicontinuous flow if and only if the collection $\left\{A_{i j k}\right\}$ is a partition of $P_{\theta}$.

It can easily be seen that this condition holds automatically for every simple binary substitution. We obtain

THEOREM 5. If $\theta$ is a binary substitution of length $r, X_{\theta}$ is either an almost automorphic flow or an $\mathrm{AI}$ extension of the equicontinuous flow $\left(\mathrm{Z}^{r}, T\right)$.

THEOREM 6. If $\theta$ is simple, and $r$ and $b$ are both prime, $x_{\theta}$ is an AI flow if and only if the collection $\left\{A_{i j k}\right\}$ is a partition of $P_{\theta}$.

By Theorem 6, we obtain a class of point-distal flows with a residual set of distal points which are not AI flows. This is significant in the light of Veech's structure theorem for point-distal flows [10], according to which every point-distal flow with a residual set of distal points has an almost automorphic extension which is an AI flow. (Leonard Shapiro, in [6], has constructed examples, of a different sort, of point-distal, non-AI flows.)

EXAMPLE. Let $b=r=3 ; \theta(0)=011, \theta(1)=202, \theta(2)=120$. It can be easily verified that $\theta$ is admissible and simple and that $m(\theta)=1$. We have $A_{010} \cap A_{011}=\{20\}$, and thus, by Theorem $6, x_{\theta}$ is not an AI flow. 
We remark that for substitutions of nonconstant length (i.e., if the blocks $\theta(0), \theta(1), \cdots, \theta(b-1)$ are not of the same length), the situation is substantially different. $X_{\theta}$ is no longer point-distal in general, and for certain $\theta, x_{\theta}$ can be shown to be weakly mixing. We hope to discuss this at greater length in a later paper.

\section{REFERENCES}

1. W. H. Gottschalk, Substitution minimal sets, Trans. Amer. Math. Soc. 109 (1963), 467-491. MR 32 \#8325.

2. W. H. Gottschalk and G. A. Hedlund, Topological dynamics, Amer. Math. Soc. Colloq. Publ., vol. 36, Amer. Math. Soc., Providence, R. I., 1955. MR 17, 650.

3. M. Keane, Generalized Morse sequences, Z. Wahrscheinlichkeitstheorie und Verw. Gebiete 10 (1968), 335-353. MR 39 \#406.

4. Harvey B. Keynes, The proximal relation in a class of substitution minimal sets, Math. Systems Theory 1 (1967), 165-174. MR 35 \#997.

5. M. Morse, Recurrent geodesics on a surface of negative curvature, Trans. Amer. Math. Soc. 22 (1921), 84-100.

6. Leonard Shapiro, Distal and proximal extensions of minimal flows (preprint).

7. W. A. Veech, Almost automorphic functions on groups, Amer. J. Math. 87 (1965), 719-751. MR 32 \#4469.

8. - Strict ergodicity in zero dimensional dynamical systems and the KroneckerWeyl theorem mod 2, Trans. Amer. Math. Soc. 140 (1969), 1-33. MR 39 \#1410.

9. - Minimal transformation groups with distal points, Bull. Amer. Math. Soc. 75 (1969), 481-486.

10. - Point-distal flows, Amer. J. Math. 92 (1970), 205-242.

Rice University, Houston, Texas 77001 